\title{
ON DETERMINING A RIEMANNIAN MANIFOLD FROM THE DIRICHLET-TO-NEUMANN MAP
}

\author{
BY MATTI LASSAS ${ }^{1}$ AND GUNTHER UHLMANN ${ }^{2}$
}

\begin{abstract}
We study the inverse problem of determining a Riemannian manifold from the boundary data of harmonic functions. This problem arises in electrical impedance tomography, where one tries to find an unknown conductivity inside a given body from voltage and current measurements made at the boundary of the body. We show that one can reconstruct the conformal class of a smooth, compact Riemannian surface with boundary from the set of Cauchy data, given on a non-empty open subset of the boundary, of all harmonic functions. Also, we show that one can reconstruct in dimension $n \geqslant 3$ compact real-analytic manifolds with boundary from the same information. We make no assumptions on the topology of the manifold other than connectedness.
\end{abstract}

○ 2001 Éditions scientifiques et médicales Elsevier SAS

RÉSUMÉ. - On étudie la détermination d'une variété riemannienne à partir des valeurs au bord de ses fonctions harmoniques. Ce problème apparait dans la tomographie d'impédance électrique, dont le but est de trouver une conductivité inconnue dans un corps à partir des mesures de voltage et de courant sur la frontière de ce corps. On démontre que la classe conforme d'une surface de Riemann compacte lisse peut être reconstruite à partir des données de Cauchy de toutes les applications harmoniques sur un sous-ensemble non vide et ouvert de la frontière. On démontre aussi qu'en dimension $n \geqslant 3$ les variétés compactes analytiques avec bord peuvent être reconstruites à partir de la même information. La seule hypothèse topologique sur les variétés est qu'elles sont connexes.

๑ 2001 Éditions scientifiques et médicales Elsevier SAS

\section{Introduction}

In this paper we study the inverse problem of determining a $n$-dimensional, $\mathrm{C}^{\infty}$-smooth, connected, compact, Riemannian manifold with boundary $(M, g)$ from the set of Cauchy data of harmonic functions given on $\Gamma$, an open non-empty subset of the boundary. More precisely, let $f \in \mathrm{C}^{\infty}(\partial M)$ with $\operatorname{supp}(f) \subset \Gamma$. Let $u \in \mathrm{C}^{\infty}(\bar{M})$ be the solution of

$$
\left\{\begin{array}{l}
\Delta_{g} u=0 \quad \text { in } M, \\
\left.u\right|_{\partial M}=f .
\end{array}\right.
$$

\footnotetext{
${ }^{1}$ Supported by Finnish Academy.

${ }^{2}$ Partially supported by NSF Grant DMS-9705792 and a grant from the Royalty Research Fund at the University of Washington.
} 
We assume that we know the Cauchy data on $\Gamma$ of all possible solutions of (1.1), or, equivalently, the Dirichlet-to-Neumann mapping

$$
\Lambda_{g, \Gamma}:\left.f \longmapsto \partial_{\nu} u\right|_{\Gamma}
$$

where $\partial_{\nu}$ is the exterior normal derivative of $u$ and $f \in \mathrm{C}_{0}^{\infty}(\Gamma)$. In this paper we address the question: is it possible to determine $(M, g)$ by knowing a non-empty open subset of the boundary $\Gamma \subset \partial M$ as a differentiable manifold and the boundary operator $\Lambda_{g, \Gamma}$ ?

This problem arises in Electrical Impedance Tomography (EIT). The question in EIT is whether one can determine the (anisotropic) electrical conductivity of a medium $\Omega$ in Euclidean space by making voltage and current measurements at the boundary of the medium. Calderón proposed this problem [3] motivated by geophysical prospection. EIT has been proposed more recently as a valuable diagnostic tool since tissues in the human body have quite different electrical conductivities. The electrical conductivity in an open subset $\Omega$ of $\mathbb{R}^{n}$ is represented by a positive definite matrix $\gamma=\left(\gamma^{i j}\right)$. The Dirichlet-to-Neumann map is the voltage to current map, that maps a voltage potential at the boundary of the medium to the induced current flux at the boundary of the medium. Note that in the study of EIT the usual metric of $\mathbb{R}^{n}$ plays usually a crucial role. We recall now some previous results on the problem for the case of a Riemannian manifold $(M, g)$ and for the EIT problem in Euclidean space. In all the cited results below $\Gamma$ is the whole boundary and we denote $\Lambda_{g}=\Lambda_{g, \partial M}$. In [7], it is observed that in dimension $n \geqslant 3$ the EIT problem is equivalent to the problem of determining a Riemannian metric $g$ from $\Lambda_{g}$ with $g_{i j}=\left(\operatorname{det} \gamma^{k l}\right)^{1 /(n-2)}\left(\gamma^{i j}\right)^{-1}$. Let us denote the closure of $\Omega$ by $\bar{\Omega}$. Then, if $\psi: \bar{\Omega} \rightarrow \bar{\Omega}$ is a diffeomorphism which is the identity at the boundary, $\Lambda_{\psi^{*} g}=\Lambda_{g}$. The natural conjecture is that this is the only obstruction to unique identifiability of the Riemannian metric (see Conjecture A in [7], p. 1098).

For isotropic metrics in $\mathbb{R}^{n}$ (i.e. $g_{i j}=\alpha(x) \delta_{i j}$ with $\delta_{i j}$ the Krönecker delta and $\alpha$ a positive function) the conjecture in dimension $n \geqslant 3$, is that the metric can be identified uniquely from the Dirichlet-to-Neumann map. This was proved for smooth isotropic metrics $g_{i j}$ in $\Omega \subset \mathbb{R}^{n}$, $n \geqslant 3$ in [11] and for $\mathrm{C}^{3 / 2}$ isotropic metrics in [1].

In the anisotropic case in dimension $n \geqslant 3$ Lee and Uhlmann proved in [7] that the conjecture is valid for simply connected real-analytic Riemannian manifolds with boundary which are in addition geodesically convex.

In the two-dimensional case, since the Laplace-Beltrami operator is conformally invariant, we have that if $\psi$ is a diffeomorphism of $\bar{\Omega}$ which is the identity at the boundary then $\Lambda_{\alpha \psi^{*} g}=\Lambda_{g}$ for all smooth functions $\alpha$ having boundary value one on $\partial M$. The natural conjecture is that this is the only obstruction to unique identifiability of the metric (see Conjecture B in [7], p. 1099). Lee and Uhlmann proved in [7] that this is the case for simply connected domains of Euclidean space. We remark that the EIT problem for domains of Euclidean space was solved in two dimensions by $A$. Nachman in [8] for $\mathrm{C}^{2}$-conductivities for domains $\Omega \subset \mathbb{R}^{2}$. This was extended to Lipschitz conductivities in [2]. The problem for anisotropic conductivities can be reduced to the case of isotropic ones by using an analog of isothermal coordinates as observed in [10].

In this paper we extend the results mentioned above of [7] in two directions. First, we assume that the Dirichlet-to-Neumann map is measured only on part of the boundary which is assumed to be real-analytic in the case $n \geqslant 3$ and $\mathrm{C}^{\infty}$-smooth in the two-dimensional case. Second we do not assume any condition on the topology of the manifold besides connectedness. Furthermore, we do not assume that the manifold is geodesically convex or that $\partial M$ or $\Gamma \subset \partial M$ are connected.

Throughout the paper we assume that one of the following conditions is satisfied:

(i) $M$ is a connected Riemannian surface; 
(ii) $n \geqslant 3$ and $(M, g)$ is a connected real analytic Riemannian manifold and the boundary $\partial M$ is real analytic in the non-empty open set $\Gamma \subset \partial M$.

Before stating the results, we explain what we mean by the reconstruction of a Riemannian manifold $(M, g)$. Since a manifold is an 'abstract' collection of coordinate patches our objective is to construct a representative of an equivalence class of the set of isometric Riemannian manifolds to $(M, g)$ or a metric space $X$ which is isometric to $(M, g)$.

In the two-dimensional case the inverse problem cannot be uniquely solved for the following reason. Assume that we have two Riemannian surfaces $(M, g)$ and $(M, \tilde{g})$ with the metrics $g$ and $\tilde{g}$ in the same conformal class, i.e. $g=g_{i j}(x)$ and $\tilde{g}=\sigma(x) g_{i j}(x), \sigma \in \mathrm{C}^{\infty}(M),\left.\sigma\right|_{\Gamma}=1, \sigma>0$. Since

$$
\Delta_{\sigma g} u=\sigma^{-1} \Delta_{g} u
$$

we see that the solutions of equations (1.1) corresponding to the metrics $g$ and $\tilde{g}$ coincide, as well as the mappings $\Lambda_{g, \Gamma}$ and $\Lambda_{\tilde{g}, \Gamma}$. However, it turns out that this is the only source of nonuniqueness. Our main result is:

THEOREM 1.1. - Assume that condition (i) or (ii) is satisfied. Then:

(i) for $\operatorname{dim} M=2$ the $\Lambda_{g, \Gamma}$-mapping and $\Gamma$ determine the conformal class of the Riemannian manifold $(M, g)$;

(ii) for a real-analytic Riemannian manifold $(M, g), \operatorname{dim} M \geqslant 3$ which boundary is real

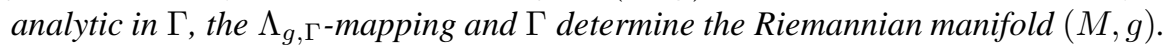

The outline of the proof for the case $\Gamma=\partial M$ is the following. Using [7] we reconstruct the metric at the boundary and enlarge $M$ to a manifold $\widetilde{M}$. For the corresponding problem in $\widetilde{M}$, using that we know the Dirichlet-to-Neumann map, we can reconstruct the Green kernels in $\widetilde{M} \backslash M$. In the two-dimensional case we use isothermal coordinates so that the Green kernels are real-analytic in these local coordinates. In dimension $n \geqslant 3$, since the manifold is real-analytic, the Green kernels are real-analytic in local coordinates. We use these Green kernels to define local coordinates. Then we continue the family of the Green kernels analytically in these coordinates. More precisely, we consider the sheaf $\mathcal{A}$ of sequences of real-analytic functions on $\mathbb{R}^{n}$, define an equivalence relation in this sheaf and define the space $\mathcal{B}$ of equivalence classes in $\mathcal{A}$. After this the reconstruction procedure of the manifold is very simple: Let $p \in \mathcal{B}$ be the element corresponding to the germs of the Green kernels at a point $x \in \widetilde{M} \backslash M$. Then the unknown manifold can be constructed by taking the path-connected component of $\mathcal{B}$ containing $p$.

The outline of the paper is as follows. In Section 2 we extend the metric $g$ to a manifold $\widetilde{M}$ so that the Green kernels are real-analytic in $\widetilde{M}$ in appropriate coordinates. In Section 3 we show that the Green kernels can be used as local coordinates and in Section 4 we introduce some sheaves in order to obtain a maximal analytic continuation of the Green kernels. In Section 5 we continue analytically the Green kernels, reconstruct the manifold as a path connected component of $\mathcal{B}$, and we prove the main result. In Section 6 we discuss some possible extensions of the results.

\section{Construction of the metric on the boundary and continuation of the manifold}

We assume first that $\Gamma=\partial M$ and introduce later the modifications for the case when the Cauchy data is given on a part of the boundary. Near $\partial M$ we use the boundary normal coordinates $(s, h)$ where $s \in \partial M$ is the point nearest to $x$ and $h=\operatorname{dist}(x, s)$. Let $\xi=\xi(s)$ be local coordinates of $\partial M$ near a given boundary point $s_{0} \in \partial M$. Thus near $s_{0}$ we have in 
$M$ coordinates $(\xi, h) \in \mathbb{R}^{n-1} \times \mathbb{R}_{+}$. In these coordinates we represent the metric by the tensor $g_{i j}(\xi, h), i, j=1, \ldots, n$.

By results of [7] (pp. 1105-1106), the operator $\Lambda_{g}$ determines in the two-dimensional case the tangential component $g_{00}(\xi, 0)$ of the metric tensor $g$ on the boundary. Correspondingly, in the case of the real-analytic manifold $M, n \geqslant 3, \Lambda_{g}$ determines all the normal derivatives $\frac{\partial^{k}}{\partial h^{k}} g_{i j}(\xi, 0), k \geqslant 0$, of the metric tensor at the boundary. Thus we define a manifold $\widetilde{M}$ by gluing to $M$ a boundary collar $\partial M \times]-r, 0]$ with metric described as follows. In dimension $n \geqslant 3$, when $r$ is small enough, we can continue the metric so that the new metric is real-analytic. In the case $n=2$ we use the product metric in $\partial M \times]-r, 0]$, which gives us a Lipschitz metric on the manifold $\widetilde{M}$, that is, $\widetilde{M}$ has $\mathrm{C}^{\infty}$-coordinates for which the metric tensor satisfies $g_{i j} \in \mathrm{C}^{0,1}(\widetilde{M})$. We denote the new metric of $\widetilde{M}$ also by $g$. Next, let

$$
U \subset \widetilde{M} \backslash \bar{M}
$$

be the closure in $\widetilde{M}$ of an open neighborhood of $\partial \widetilde{M}$ (see Fig. 1). We will use later a family of open neighborhoods $U_{r} \subset \widetilde{M} \backslash M$,

$$
U_{r}=\{x \in \widetilde{M}: \mathrm{d}(x, U)<r\}, \quad 0<r<r_{0},
$$

where $r_{0}>0$ is small enough.

We use the Green functions of the Laplace-Beltrami operator which are solutions of the equation

$$
\left\{\begin{array}{l}
\Delta_{g} G(\cdot, y)=\delta_{y} \quad \text { in } \widetilde{M}, \\
\left.G(\cdot, y)\right|_{\partial \widetilde{M}}=0,
\end{array}\right.
$$

where $y \in \widetilde{M}$. As usual, we denote by $\delta_{y}$ the Dirac distribution which satisfies $\left(\delta_{y}, f\right)_{\mathrm{L}^{2}(M, g)}=$ $f(y)$. In the following we consider $y$ as a parameter and use the notation

$$
h_{y}(x)=G(x, y)
$$

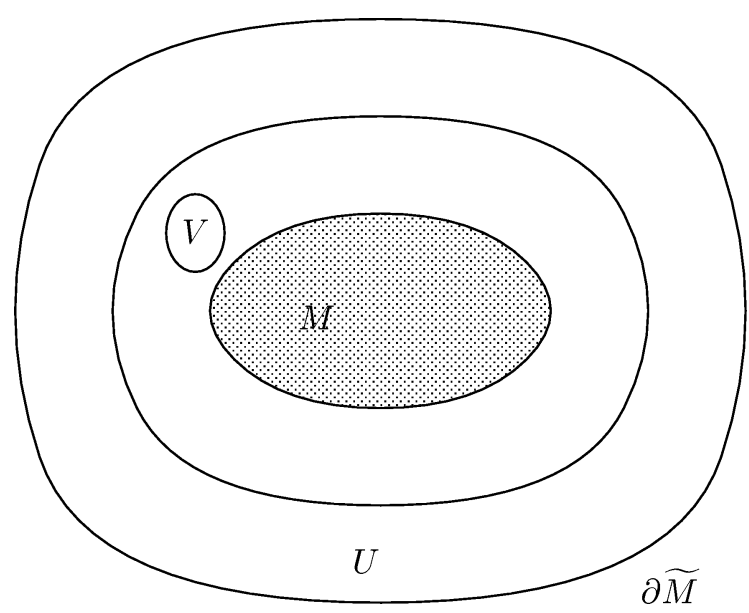

Fig. 1. Extension $\widetilde{M}$ of $M$, the neighborhood $U$ of $\partial \widetilde{M}$ and the neighborhood $V$ where the continuation procedure is started ( $V$ will be defined in Section 5 ). 
LEMMA 2.1. - Every point $x \in \widetilde{M} \backslash U$ has a coordinate neighborhood $(W, \phi), \phi: W \rightarrow \mathbb{R}^{n}$ where the Green functions $h_{y} \circ \phi^{-1}, y \in U$, are real-analytic.

Proof. - For the real-analytic $\widetilde{M}, n \geqslant 3$ by definition, each point has a neighborhood with real-analytic coordinate function $\phi$. Since the metric is real-analytic and the Green functions $h_{y}(x), x \neq y$ satisfy the Laplace-Beltrami equation which is a differential equation with realanalytic coefficients, it follows by [4] (Theorem 9.5.1) that $h_{y}(x)$ are real-analytic functions in the $\phi$-coordinates.

In the two-dimensional case the metric of $\widetilde{M}$ is Lipschitz. Then every (interior) point $x \in \widetilde{M}$ has a neighborhood $W$ with isothermal coordinates

$$
\phi(x)=\left(\phi_{1}(x), \phi_{2}(x)\right), \quad \phi \in \mathrm{C}^{1, \alpha}, \alpha<1,
$$

such that the metric tensor is of the form:

$$
g_{i j}(x)=\sigma(x) \delta_{i j}, \quad \sigma(x)>0
$$

(see [16], Sections II.2 and II.6.1, or alternatively, [10], Lemma 2.2 and [16], Theorem 2.1). In these $\mathrm{C}^{1, \alpha}$-coordinates the Laplace-Beltrami operator is well defined in weak sense, and particularly the formula (1.2) is valid. Thus we obtain

$$
\Delta\left(h_{y} \circ \phi^{-1}\right)(\phi(x))=\sigma(x) \Delta_{g} h_{y}(x)=0 \quad \text { for } y \notin W,
$$

where $\Delta=\partial_{1}^{2}+\partial_{2}^{2}$ is the Euclidean Laplacian. This implies that $h_{y} \circ \phi^{-1}$ is harmonic in $\mathbb{R}^{2}$ with respect to the Euclidean metric. Thus $h_{y}$ is real-analytic in the $\phi$-coordinates.

Our first goal is to determine Green's functions in the boundary layer.

Lemma 2.2. - The Dirichlet-to-Neumann map on $\partial M$ and the metric $g$ in $\widetilde{M} \backslash M$ determine the values of Green's functions $h_{y}(x)$ for $x, y \in \widetilde{M} \backslash M$.

Proof. - Let $y \in \widetilde{M} \backslash M$ and $u$ be a function in $\widetilde{M} \backslash M$. Then $u$ has a continuation $u_{1}$ to $\widetilde{M}$ which satisfies the equation

$$
\left\{\begin{array}{l}
\Delta_{g} u_{1}=\delta_{y} \\
\left.u_{1}\right|_{\partial \widetilde{M}}=0
\end{array} \text { in } \widetilde{M},\right.
$$

if and only if

$$
\left\{\begin{array}{l}
\Delta_{g} u=\delta_{y} \\
\left.\partial_{\nu} u\right|_{\partial M}=\Lambda_{g}\left(\left.u\right|_{\partial M}\right), \\
\left.u\right|_{\partial \widetilde{M}}=0,
\end{array} \quad \text { in } \widetilde{M} \backslash M,\right.
$$

where $\nu$ is the unit normal vector of $\partial M$ pointing towards $\widetilde{M} \backslash M$. In other words, (2.8) means that $u$ solves Eq. (2.7) in $\widetilde{M} \backslash M$ and its Cauchy data coincides with the Cauchy data of some solution in $M$. Thus we can consider (2.7) and (2.8) as equivalent equations.

Since we know the metric $g$ in $\widetilde{M} \backslash M$ and we know the Dirichlet-to-Neumann map $\Lambda_{g}$, we can uniquely solve (2.8). Since this solution has an extension to $\widetilde{M}$ solving (2.7), we have determined $h_{y}(x)$ for $x, y \in \widetilde{M} \backslash M$. 


\section{Properties of Green's functions}

First we show that the values of Green's functions at $x$ determine $x$ uniquely.

LEMMA 3.1. - The mapping

$$
x \in \widetilde{M} \longmapsto\left(h_{y}(x)\right)_{y \in U}
$$

is injective.

Proof. - Assume that $G(x, y)=G\left(x^{\prime}, y\right)$ for all $y \in U$. By the symmetry of the Green kernel, $G(y, x)=G\left(y, x^{\prime}\right)$ for all $y \in U$. By the unique continuation principle, this holds for all $y \in \widetilde{M} \backslash\left\{x, x^{\prime}\right\}$. Moreover, $G(x, y)$ is at least $\mathrm{C}^{1, \alpha}$-smooth when $x \neq y$ and when $y$ is near to a given $x$ it has the asymptotics ([12], Section 7.11 using the coordinates (2.5) in the case $n=2$ )

$$
\begin{cases}G(y, x)=c_{n} \mathrm{~d}(y, x)^{2-n}+\mathcal{O}\left(\mathrm{d}(y, x)^{3-n}\right), & n \geqslant 3 \\ G(y, x)=c_{n} \log \mathrm{d}(y, x)+\mathcal{O}(1), & n=2\end{cases}
$$

where the constants $c_{n} \neq 0$ depend only on $n$ and $d$ is the distance in $\widetilde{M}$. Thus for given $x$, $G(y, x)$ is singular at $y=x$ and at this point only. Similarly, $G\left(y, x^{\prime}\right)$ is singular at the unique point $y=x^{\prime}$ which implies that $x=x^{\prime}$.

We denote by $\mathrm{S} \widetilde{M}$ the sphere bundle of vectors $(x, \xi) \in T \widetilde{M},|\xi|=1$ and we use the notation $\mathrm{d}_{x} h=\left.\mathrm{d} h\right|_{x}$ for differential of $h$ at $x$. In the following two lemmas, $\left.r \in\right] 0, r_{0}[$ is a fixed number.

LEMMA 3.2. - There is $c_{0}>0$ such that for any $(x, \xi) \in \mathrm{S}\left(\widetilde{M} \backslash U_{r}\right)$ there is $y \in U$ such that

$$
\left|\mathrm{d}_{x} h_{y}(\xi)\right| \geqslant c_{0}
$$

Proof. - In the case $n \geqslant 3$ the Riemannian manifold is a real-analytic. Thus for a given $(x, \xi) \in \mathrm{S}\left(\widetilde{M} \backslash U_{r}\right)$ there is a real-analytic path $\gamma:\left[0, t_{1}\right] \rightarrow M$ such that

$$
\gamma(0)=x, \quad \dot{\gamma}(0)=\xi, \quad \gamma\left(t_{1}\right) \in U^{\mathrm{int}} .
$$

Correspondingly, in the case $n=2$, let $\gamma_{1}$ be a smooth simple path from $y \in U^{\text {int }}$ to $x$ and $V$ be a small neighborhood of the path $\gamma_{1}$. Since $\widetilde{M}$ is a $\mathrm{C}^{\infty}$-manifold, we can consider $V$ as a coordinate neighborhood $(V, \psi)$ with a coordinate function $\psi \in \mathrm{C}^{\infty}$. For instance, when $V$ is small enough, we can define tubular coordinates in $V$. Then $\gamma_{1}$ has a neighborhood $V_{1} \subset V$ on which we can construct isothermal coordinates $\phi$ satisfying (2.5) and (2.6). Indeed, if $g_{i j}(z)$, $z \in \psi(V)$ is the metric tensor in the $\psi$-coordinates, there are functions $f_{i j} \in \mathrm{C}^{0,1}\left(\mathbb{R}^{2}\right)$ which coincide with $g_{i j}(z)$ in $\psi\left(V_{1}\right)$ and with $\delta_{i j}$ in $\mathbb{R}^{2} \backslash \psi(V)$. The existence of the isothermal coordinates near $\gamma_{1}$ follows then from [16] (see Section II.2 and Theorem 2.12). Indeed, by solving a Beltrami equation in $\mathbb{R}^{2}$ corresponding to the metric $f_{i j}$, we can construct in $V_{1}$ coordinates in which $g_{i j}$ has the form (2.6). In these isothermal coordinates the Green functions are real-analytic. By using the Weierstrass theorem, we find in these coordinates a real-analytic path $\gamma$ which approximates $\gamma_{1}$ and satisfies conditions (3.2).

Thus in both cases $n=2$ and $n \geqslant 3$ we can define a real-analytic function

$$
b: t \longmapsto \mathrm{d}_{x} h_{\gamma(t)}(\xi), \quad t \in\left[0, t_{1}\right] .
$$


Assume that this function vanishes identically for all $t$ such that $\gamma(t) \in U$. Since $U^{\text {int }}$ is an open set, $b$ vanishes identically and in particular near $t=0$. However, the function $G(x, y)$ has asymptotics (3.1). This is a contradiction if $b$ vanishes for all $t$ near zero. Thus, for every $(x, \xi)$,

$$
H(x, \xi)=\sup _{y \in U}\left|\mathrm{~d}_{x} h_{y}(\xi)\right|>0 .
$$

The claim follows since $H$ is continuous in $\mathrm{S}\left(\widetilde{M} \backslash U_{r}\right)$ which is a compact set.

Next, we denote $\mathrm{B}(x, r)=\left\{x^{\prime} \in \widetilde{M}: \mathrm{d}\left(x^{\prime}, x\right) \leqslant r\right\}$.

LEMMA 3.3. - There exist points $\tilde{y}_{1}, \ldots, \tilde{y}_{p} \in U$ such that

$$
Q: \widetilde{M} \backslash U_{r} \longrightarrow \mathbb{R}^{p}, \quad x \longmapsto\left(h_{\tilde{y}_{k}}(x)\right)_{k=1}^{p}
$$

is bi-Lipschitz mapping to its image. In particular, $Q$ is injective.

Proof.-Obviously, for any $\tilde{y}_{1}, \ldots, \tilde{y}_{p}$ the mapping $Q$ is Lipschitz. Next, we show the existence of $\tilde{y}_{1}, \ldots, \tilde{y}_{p}$ such that $Q$ is injective and its left inverse is Lipschitz.

Let $x \in \widetilde{M} \backslash U_{r}$. Then there are points $y_{1}=y_{1}(x), \ldots, y_{n}=y_{n}(x) \in U$ such that the vectors $\mathrm{d}_{x} h_{y_{j}}, j=1, \ldots, n$, are linearly independent in $\mathrm{T}_{x}^{*} \widetilde{M}$. Indeed, in the opposite case we obtain a contradiction with Lemma 3.1 when $\xi$ is a vector vanishing in the space spanned by $\mathrm{d}_{x} h_{y}$, $y \in U$. Since $h_{y_{j}}$ are at least $\mathrm{C}^{1, \alpha}$-smooth, it follows from the inverse function theorem that the function $z \mapsto\left(h_{y_{j}}\right)_{j=1}^{n}$ has a local inverse function which is $\mathrm{C}^{1, \alpha}$-smooth. Hence there is $\rho_{x}>0$ such that for $x^{\prime}, x^{\prime \prime} \in \mathrm{B}\left(x, 2 \rho_{x}\right)$

$$
C_{1} \mathrm{~d}\left(x^{\prime}, x^{\prime \prime}\right) \leqslant\left\|\left(h_{y_{j}}\left(x^{\prime}\right)-h_{y_{j}}\left(x^{\prime \prime}\right)\right)_{j=1}^{n}\right\|_{\mathbb{R}^{n}} \leqslant C_{2} \mathrm{~d}\left(x^{\prime}, x^{\prime \prime}\right),
$$

where $C_{1}, C_{2}>0$. Since $\widetilde{M} \backslash U_{r}$ is compact, we can choose a finite cover of balls $\mathrm{B}\left(x_{\ell}, \rho_{x_{\ell}}\right)$, $\ell=1, \ldots, L$, such that (3.3) is valid with uniform $C_{1}$ and $C_{2}$. Let $\rho=\min \rho_{x_{\ell}}$. Moreover, by using the compactness of $\widetilde{M} \backslash U_{r}$ and Lemma 3.1 we can choose points $z_{1}, \ldots, z_{m}$ and $\varepsilon>0$ such that

$$
\left\|\left(h_{z_{j}}\left(x^{\prime}\right)-h_{z_{j}}\left(x^{\prime \prime}\right)\right)_{j=1}^{m}\right\|_{\mathbb{R}^{m}} \geqslant \varepsilon
$$

when $\mathrm{d}\left(x^{\prime}, x^{\prime \prime}\right) \geqslant \rho$. Thus by choosing

$$
\left\{\tilde{y}_{k}: k=1, \ldots, p\right\}=\left\{y_{j}\left(x_{\ell}\right): j=1, \ldots, n, \ell=1, \ldots, L\right\} \cup\left\{z_{j}: j=1, \ldots, m\right\}
$$

we prove the claim.

\section{Sheaf of families of analytic functions}

In this section we do the preliminary work so that we can use real-analytic continuations of the Green functions in the next section. We first explain informally the ideas on how the construction of a manifold from the Dirichlet-to-Neumann map can be done in the two-dimensional case.

The basic idea can be seen in Fig. 2. For every fixed $x \in M$ there is a neighborhood $W$ of $x$ and points $y_{1}, y_{2} \in U$ such that $h_{y_{1}}$ and $h_{y_{2}}$ form regular coordinates

$$
H: W \longrightarrow \mathbb{R}^{2}, \quad z \longmapsto\left(h_{y_{1}}(z), h_{y_{2}}(z)\right) \text {. }
$$

Moreover, in $W$ we have isothermal coordinates $\phi: W \rightarrow \mathbb{R}^{2}$. Then the functions $h_{y_{1}} \circ \phi^{-1}$ and $h_{y_{2}} \circ \phi^{-1}$ are real-analytic. Hence by the inverse function theorem the mapping

$$
\phi \circ H^{-1}:\left(h_{y_{1}}(z), h_{y_{2}}(z)\right) \longmapsto \phi(z)
$$




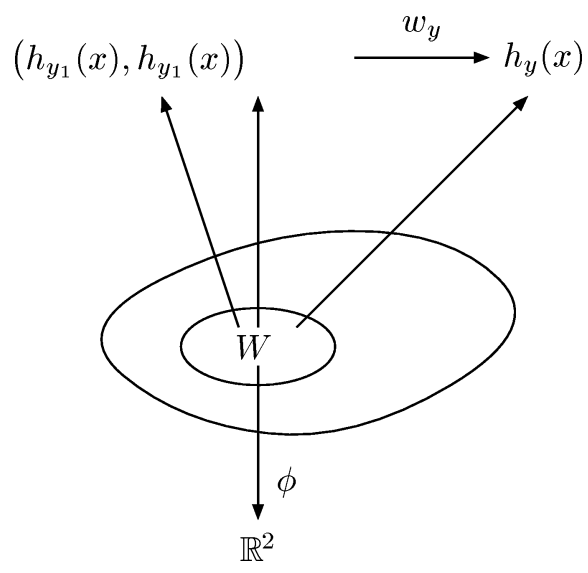

Fig. 2. Isothermal coordinates $\phi$ on a coordinate neighborhood $W$ and the Green functions $h_{y}$.

is real analytic in the neighborhood $H(W) \subset \mathbb{R}^{2}$ of $\left(h_{y_{1}}(x), h_{y_{2}}(x)\right)$. Let $y \in U$ be an arbitrary point. Since $h_{y} \circ \phi^{-1}: \phi(W) \rightarrow \mathbb{R}$ and $\phi \circ H^{-1}: H(W) \rightarrow \mathbb{R}^{2}$ are real analytic, we see that also their composition $w_{y}=h_{y} \circ H^{-1}: H(W) \rightarrow \mathbb{R}$,

$$
w_{y}:\left(h_{y_{1}}(z), h_{y_{2}}(z)\right) \longmapsto h_{y}(z)
$$

is real analytic (see Fig. 2). In other words, the Green functions corresponding to points $y_{1}$ and $y_{2}$ form coordinates in which all other Green's functions $h_{y}$ are real-analytic. Hence all the Green functions $h_{y}(y \in U)$ can be analytically continued as functions of the given Green functions $h_{y_{1}}(x)$ and $h_{y_{2}}(x)$ as long as these Green functions form a coordinate system. When Green functions $h_{y_{1}}(x)$ and $h_{y_{2}}(x)$ do not form any more regular coordinates, we choose new points $y_{1}^{\prime}$ and $y_{2}^{\prime}$, use the corresponding Green functions as coordinates, and continue analytically the other Green functions. This procedure can be repeated arbitrarily many times until the constructed neighborhoods cover the whole manifold. In other words, we continue the Green functions analytically as function of themselves and show that the maximal analytic continuation is equivalent to the desired manifold which we want to construct.

To make this approach rigorous we analyze the maximal real-analytic continuation by applying the theory of sheaves which is very useful in going from local information to global information.

Now, we return to rigorous analysis in dimension $n=\operatorname{dim}(M) \geqslant 2$. Since $U$ is compact, for any $r_{1}>0$ there is $m<\infty$ with

$$
m=m\left(r_{1}\right)=\max \left\{\left|h_{y}(x)\right|: y \in U, x \in \partial U_{r_{1}}\right\} .
$$

By (3.1), $m\left(r_{1}\right) \rightarrow \infty$ when $r_{1} \rightarrow 0$. We define the set

$$
K_{m}=\left\{x \in \widetilde{M} \backslash U:\left|h_{y}(x)\right|<m \text { for all } y \in U\right\} .
$$

Since $U$ is compact, for any $x \notin U$ the function $y \mapsto h_{y}(x)$ attains its maximum at some $y$. Since the differentials $\mathrm{d}_{x} h_{y}$ are uniformly bounded when $y \in U$ and $\mathrm{d}(x, U)>\varepsilon$, we see that $K_{m}$ is open. Moreover, when $x \in \partial K_{m}$, this shows that there is $y \in U$ such that

$$
h_{y}(x)=m \text {. }
$$

When $m$ is large enough, $M \subset K_{m}$. Thus we can define:

$$
\text { let } M_{m} \text { be the connected component of } K_{m} \text { containing } M \text {. }
$$


Next we define the sheaf which is needed for maximal real-analytic continuation (for the standard definitions and properties of sheaves, we refer to [14]). As usual, the maximal analytic continuation is a connected component of the sheaf of analytic functions.

We denote by $S$ a pair $\left(\left(f_{y}\right)_{y \in U}, \omega\right)$ corresponding to the family of real-analytic functions $\left.f_{y}: \omega \rightarrow\right]-m, m\left[\right.$ which are indexed by $y \in U$ and defined in a domain $\omega \subset \mathbb{R}^{n}, n=\operatorname{dim}(M)$. For instance, in the case $n=2$ the Green functions $w_{y}: H(W) \rightarrow \mathbb{R}$ given in formula (4.1) define the pair $\left(\left(w_{y}\right)_{y \in U}, \omega\right)$ with $\omega=H(W)$.

These pairs define a pre-sheaf $\mathcal{H}=\left(\mathcal{H}_{\omega}, \rho_{\omega^{\prime}, \omega}\right)$ where

$$
\mathcal{H}_{\omega}=\left\{\left(\left(f_{y}\right)_{y \in U}, \omega\right): f_{y}: \omega \rightarrow \mathbb{R} \text { are real-analytic, }\left|f_{y}(z)\right|<m, z \in \omega\right\} .
$$

Here $\omega \subset \mathbb{R}^{n}$ are open connected sets and $\rho_{\omega^{\prime}, \omega}, \omega^{\prime} \subset \omega$ are the restriction mappings $\rho_{\omega^{\prime}, \omega}:\left(f_{y}\right) \mapsto\left(\left.f_{y}\right|_{\omega^{\prime}}\right), \rho_{\omega^{\prime}, \omega}: H_{\omega} \rightarrow H_{\omega^{\prime}}$.

Let $\mathcal{S}$ be the associated sheaf corresponding to $\mathcal{H}$ with stalks $\mathcal{S}_{z}, z \in \mathbb{R}^{n}$. We recall that the sheaf $\mathcal{S}$ is the disjoint union of stalks $\mathcal{S}_{z}$ and the elements of $\mathcal{S}_{z}$, called germs, are the direct limits

$$
s=\lim _{\rightarrow}\left(\left(f_{y}\right)_{y \in U}, \omega\right)=\lim _{\omega \searrow\{z\}}\left(\left(f_{y}\right)_{y \in U}, \omega\right) .
$$

Roughly speaking, $s$ corresponds to the Taylor expansions of the functions $f_{y}$ at $z$. We define the natural projections

$$
\pi: \mathcal{S} \longrightarrow \mathbb{R}^{n}, \quad s \longmapsto z \text { for } s \in \mathcal{S}_{z}
$$

and

$$
\rho_{z, \omega}: \mathcal{H}_{\omega} \longrightarrow \mathcal{S}_{z}, \quad S=\left(\left(f_{y}\right)_{y \in U}, \omega\right) \longmapsto s=\lim _{\omega^{\prime} \backslash\{z\}}\left(\left(f_{y}\right)_{y \in U}, \omega^{\prime}\right),
$$

where $z \in \omega$. We recall that the usual sheaf topology in $\mathcal{S}$ is the topology generated by the sets

$$
Y_{S, \omega}=\left\{\rho_{z, \omega}(S) \in \mathcal{S}: z \in \omega\right\}
$$

corresponding to an open domain $\omega \subset \mathbb{R}^{n}$ and a pair $S=\left(\left(f_{y}\right), \omega\right) \in \mathcal{H}_{\omega}$.

We define the evaluation mappings

$$
\mathcal{E}: \mathcal{S} \longrightarrow \mathbb{R}^{U}, \quad s=\rho_{z, \omega}\left(\left(f_{y}\right)_{y \in U}, \omega\right) \in \mathcal{S}_{z} \longmapsto\left(f_{y}(z)\right)_{y \in U},
$$

and $\mathcal{E}^{y}: s \mapsto f_{y}(z)$. The mapping $\mathcal{E}$ is continuous where $\mathbb{R}^{U}$ has the usual product topology.

Next we do the constructions which enables us to use the Green functions as coordinates. For this, let us define

$$
H^{\vec{y}}: \widetilde{M} \longrightarrow \mathbb{R}^{n}, \quad x \longmapsto\left(h_{y_{j}}(x)\right)_{j=1}^{n},
$$

where $\vec{y}=\left(y_{1}, \ldots, y_{n}\right)$ is a $n$-tuple of $n$ different points $y_{j} \in U$. We denote by $\mathcal{Y}$ the set of $\vec{y}$ 's and use the notation $H=H^{\vec{y}}$ when there is no danger of confusion. Assume now that $H^{\vec{y}}: W \rightarrow \mathbb{R}^{n}$ defines regular coordinates in $W \subset \widetilde{M} \backslash U$. We consider the pair $S=$ $\left(\left(w_{y}\right)_{y \in U}, \omega\right)$ where $w_{y}=h_{y} \circ\left(H^{\vec{y}}\right)^{-1}$ and $\omega=H^{\vec{y}}(W)$. In other words, $S$ corresponds to the Green functions $h_{y}$ in the $H^{\vec{y}}$-coordinates. Then for $t=\left(t_{1}, \ldots, t_{n}\right) \in H^{\vec{y}}(W) \subset \mathbb{R}^{n}$ we have $h_{y_{j}} \circ H^{-1}(t)=t_{j}$. This means that $\left(w_{y_{1}}, \ldots, w_{y_{n}}\right)$ is the identity mapping $H(W) \rightarrow H(W)$. Motivated by this observation, we define the set $\mathcal{A}^{\vec{y}} \subset \mathcal{S}$ as follows. Let

$$
\mathcal{A}^{\vec{y}}=\left\{s: s=\rho_{z, \omega}\left(\left(f_{y}\right), \omega\right) \text { where } f_{y_{j}}(t)=t_{j} \text { for } t \in \omega, j=1, \ldots, n\right\} .
$$

Also, we denote by $\mathcal{A}$ the disjoint union 


$$
\mathcal{A}=\coprod_{\vec{y} \in \mathcal{Y}} \mathcal{A}^{\vec{y}}
$$

Using the sheaf topology of $\mathcal{S}$, the topology of $\mathcal{A}$ is generated by the open sets

$$
Y_{S, \omega, \vec{y}}=\left\{s_{z}=\rho_{z, \omega}(S) \in \mathcal{A}^{\vec{y}}: z \in \omega\right\},
$$

where $\omega \subset \mathbb{R}^{n}$ is open, $S=\left(\left(f_{y}\right), \omega\right)$ and $\vec{y} \in \mathcal{Y}$.

We are going to use certain equivalence classes of the sheaf as the points of the desired reconstructed manifold. This equivalence relation reflects the fact that we can represent a manifold using different coordinate systems.

For $s_{1}, s_{2} \in \mathcal{A}$ we define the following relation: we say that $s_{1} \sim s_{2}$ if and only if there exist representations $s_{1}=\rho_{z^{\prime}, \omega^{\prime}}\left(\left(f_{y}\right), \omega^{\prime}\right), s_{2}=\rho_{z^{\prime \prime}, \omega^{\prime \prime}}\left(\left(g_{y}\right), \omega^{\prime \prime}\right)$ and there is a real-analytic diffeomorphism $F: \omega^{\prime \prime} \rightarrow \omega^{\prime}, F\left(z^{\prime \prime}\right)=z^{\prime}$ such that

$$
f_{y} \circ F=g_{y} \quad \text { for all } y \in U .
$$

Note that, if $s_{1}, s_{2} \in \mathcal{A}^{\vec{y}}, \vec{y} \in \mathcal{Y}$, then the relation $s_{1} \sim s_{2}$ yields

$$
\left.f_{y}\right|_{\omega^{\prime} \cap \omega^{\prime \prime}}=\left.g_{y}\right|_{\omega^{\prime} \cap \omega^{\prime \prime}}, \quad y \in U .
$$

The relation $\sim$ is clearly an equivalence relation. Let us denote by $[s]$ the equivalence classes, $\mathcal{B}=\mathcal{A} / \sim$ and

$$
e: \mathcal{A} \longrightarrow \mathcal{B}, \quad s \longmapsto[s] .
$$

The set $\mathcal{B}$ has the topology co-induced by $e$ : the set $B \subset \mathcal{B}$ is open if its pre-image in $\mathcal{A}$ is open. This makes $e$ continuous and open mapping. Since for $s_{1} \sim s_{2}$ we have $\mathcal{E}\left(s_{1}\right)=\mathcal{E}\left(s_{2}\right)$, we can define the evaluation mapping $\mathcal{E}$ also on $\mathcal{B}$.

\section{Construction of the unknown manifold}

After the previous preparations we now realize our 'construction procedure'. In the first place, let $x \in M_{m} \backslash M$. Then by Lemma 3.2 there are $\vec{y}=\left(y_{1}, \ldots, y_{n}\right) \in \mathcal{Y}$ such that $H(x)=$ $\left(h_{y_{1}}(x), \ldots, h_{y_{n}}(x)\right)$ given in (4.7) form regular coordinates in some neighborhood $V_{1}$ of $x$. Moreover, by Lemma 2.1 there is a coordinate neighborhood $\left(V_{2}, \phi\right)$ of $x$ such that $H \circ \phi^{-1}$ and $h_{y} \circ \phi^{-1}, y \in U$, are real-analytic. Thus there is a neighborhood $V \subset V_{1} \cap V_{2}$ of $x$ where $h_{y} \circ H^{-1}$ are real-analytic for all $y \in U$. The set $V$ is where the analytic continuation is started (see Fig. 1). Secondly, let

$$
\omega=H(V), \quad z=H(x)
$$

and define a germ

$$
s_{0}=\rho_{z, \omega}\left(S_{0}\right), \quad S_{0}=\left(\left(f_{y}^{0}\right)_{y \in U}, \omega\right), \quad f_{y}^{0}=h_{y} \circ H^{-1},
$$

where $h_{y}$ are the Green functions defined in formula (2.4). So, this germ $s_{0}$ corresponds to the Green functions at $x$.

With the above preliminaries our reconstruction procedure of the unknown manifold is quite simple.

Definition 5.1. - Let $N$ be the connected component of $\mathcal{B}$ containing [ $\left.s_{0}\right]$. We call $N$ the maximal analytic continuation of $s_{0}$. 
We will show that $N$ is homeomorphic to the manifold $M_{m}$ defined in formula (4.4) and construct later an appropriate metric on $N$.

First we show that the set $N$ has a manifold structure. Since $N$ is open, any $[s] \in N$ has a neighborhood $e(Y)$, where $Y \subset \mathcal{A}^{\vec{y}}$ is as in (4.9). In the set $e(Y)$ we have coordinates

$$
\Psi:[s] \longmapsto \pi(s), \quad s \in Y .
$$

Notice that every $\vec{y}$ such that $[s] \in e\left(\mathcal{A}^{\vec{y}}\right)$ defines coordinates near $[s]$. If $[s] \in \mathrm{N}$ has two representations $s_{1} \sim s_{2}, s_{1} \in \mathcal{A}^{\vec{y}}$ and $s_{2} \in \mathcal{A}^{\vec{y}^{\prime}}$, the function $F$ given in formula (4.10) define the real-analytic transition function between the corresponding coordinate charts. Thus $N$ has a real-analytic manifold structure.

THEOREM 5.1. - There is a homeomorphism $R: M_{m} \rightarrow N$. Moreover, in $N$ the evaluation mapping $\mathcal{E}$ gives the values of the Green functions,

$$
\mathcal{E}[R(x)]=\left(h_{y}(x)\right)_{y \in U}, \quad x \in M_{m} .
$$

Proof. - The proof consists of several steps.

Step 1: for every $x \in M_{m}$ there are points $y_{1}=y_{1}(x), \ldots, y_{n}=y_{n}(x) \in U$ and $\vec{y}(x)=\vec{y}=$ $\left(y_{1}, \ldots, y_{n}\right)$ such that $H^{\vec{y}}$ forms regular coordinates in neighborhood $W$ of $x$. We denote next $H=H^{\vec{y}}$. Now the Green functions define the family

$$
S(x, \vec{y})=\left(\left(f_{y}\right)_{y \in U}, \omega\right), \quad f_{y}=h_{y} \circ H^{-1}, \quad \omega=\omega(x)=H(W) .
$$

Also, we denote $z(x)=H(x)$.

For given $\vec{y}=\left(y_{1}, \ldots, y_{n}\right)$ we consider the set of $x$ at which $H^{\vec{y}}$ defines regular coordinates. We denote this set by

$$
M^{\vec{y}}=\left\{x \in M_{m}: \operatorname{det}\left(\mathrm{d}_{x} H^{\vec{y}}\right) \neq 0\right\} .
$$

Step 2: we define $R$ to be the mapping which maps $x$ to the germ of the Green functions at $x$,

$$
R: M_{m} \longrightarrow \mathcal{B}, \quad x \longmapsto\left[\rho_{z(x), \omega(x)}(S(x, \vec{y}(x)))\right] .
$$

Obviously $R(x)$ is independent of the choice of $\vec{y}(x)$.

Next, we show that $R$ is continuous and open in $M_{m}$. Let $x^{\prime} \in W$. Then also the point $x^{\prime}$ has a neighborhood where the coordinates $H_{\vec{y}}$ corresponding to $\vec{y}=\vec{y}(x)$ are regular. Hence

$$
R\left(x^{\prime}\right)=e\left(\rho_{H\left(x^{\prime}\right), \omega(x)}(S(x, \vec{y}(x)))\right)
$$

with $e$ as in Section 4. By the definition of topology of $\mathcal{B}, R$ is continuous and open in $M_{m}$.

Step 3: next, we study the bijectivity of $R$. By Lemma 3.1, the mapping $R$ is injective. We show the surjectivity of $B$ in the next steps. We note that the connected components of $\mathcal{B}$ coincide with the path-connected components. Thus, let $[s] \in N$ and let $\gamma:[0,1] \rightarrow \mathcal{B}$ be a path from $\left[s_{0}\right]$ to $[s]$. Let

$$
K=\left\{t \in [ 0 , 1 ] : \text { for all } t ^ { \prime } \in \left[0, t\left[\text { we have } \gamma\left(t^{\prime}\right) \in R\left(M_{m}\right)\right\} .\right.\right.
$$

Obviously $K$ is a closed set. We are going to show that it is also open. Let $t_{1}=\sup K$ and $B \subset \mathcal{B}$ be a neighborhood of $\gamma\left(t_{1}\right)$. The path $\gamma$ defines a path $\mathcal{E}(\gamma(t))$ on $\mathbb{R}^{U}$. Our aim is to map this path to a path $\mu$ on the manifold $M_{m}$.

Our construction procedure of finding the maximal analytic continuation can be now interpreted as continuation along paths on $M_{m}$. However, there are two problems which can be schematically shown in the Fig. 3. In the first place, the path $\mu$ can leave $M_{m}$ as in case (i). 


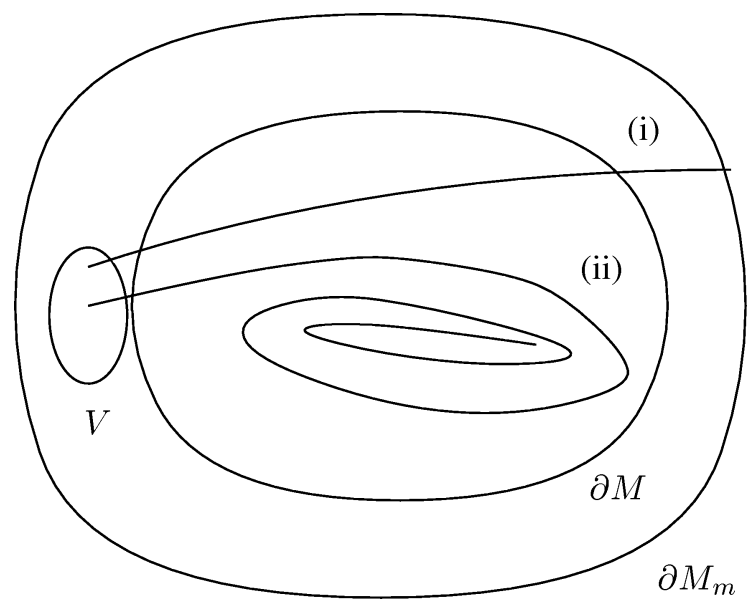

Fig. 3. Paths which can cause the wrong kind of continuations of $h_{y}$.

Secondly, as in case (ii), the path $\mu\left(\left[0, t^{\prime}[)\right.\right.$ can have infinite length. In this case the Green functions $h_{y}(\mu(t))$ might have a continuation over the point $t=t^{\prime}$ which would not correspond to the Green functions on $M_{m}$. These two problems will be dealt with below.

Step 4: we show that the path $\mu\left(\left[0, t_{1}[)\right.\right.$ has a limit point. Let $Q$ be the mapping defined in Lemma 3.3, with corresponding points $\tilde{y}_{1}, \ldots, \tilde{y}_{p}$. Next, let $P: \mathbb{R}^{U} \rightarrow \mathbb{R}^{p}$ be the projection $\left(f_{y}\right)_{y \in U} \mapsto\left(f_{\tilde{y}_{1}}, \ldots, f_{\tilde{y}_{p}}\right)$. Let

$$
\tilde{\gamma}(t)=P \mathcal{E}(\gamma(t))
$$

be a path on $\mathbb{R}^{p}$. Since $\tilde{\gamma}(t)$ is in the range of $Q$ when $t<t_{1}$, we can define a path

$$
\mu(t)=Q^{-1} P \mathcal{E}(\gamma(t)), \quad t<t_{1},
$$

on $M_{m}$. Note that now we have three corresponding paths, namely the path $\gamma$ on the sheaf $\mathcal{B}$, the path $\tilde{\gamma}$ in $\mathbb{R}^{p}$ and the path $\mu$ on $M_{m}$.

Since $P \mathcal{E} \gamma$ is continuous near $t_{1}$ and $Q$ is bi-Lipschitz, we see that $\mu$ maps Cauchy sequences of $\left[0, t_{1}\right.$ [ to Cauchy sequence of $M_{m}$. Hence there exists a limit

$$
\mu\left(t_{1}\right)=\lim _{t \rightarrow t_{1}-} \mu(t) \in \overline{M_{m}} .
$$

We denote $x_{1}=\mu\left(t_{1}\right)$.

Step 5: next, we show that $x_{1} \in M_{m}$. Since $M_{m}$ is open, it is enough to show that $x_{1} \notin \partial M_{m}$. Since $\mathcal{E} \gamma$ is continuous, we know by definition (4.5) of sets $\mathcal{H}_{\omega}$ that

$$
\left.\lim _{t \rightarrow t_{1}-} \mathcal{E}(\gamma(t))=\mathcal{E}\left(\gamma\left(t_{1}\right)\right) \in\right]-m, m\left[{ }^{U}\right.
$$

exists. However, if $x_{1} \in \partial M_{m}$, by formula (4.3) there is $y$ such that $\left|h_{y}\left(x_{1}\right)\right|=m$. Then we would have

$$
\lim _{t \rightarrow t_{1}-}\left|\mathcal{E}^{y}(\gamma(t))\right|=\lim _{t \rightarrow t_{1}-}\left|h_{y}(\gamma(t))\right|=m
$$

which is a contradiction with (5.9). Thus $x_{1} \in M_{m}$ and $\gamma\left(t_{1}\right)=R\left(x_{1}\right) \in R\left(M_{m}\right)$. This means that the case (i) in Fig. 3 cannot happen.

Step 6: next, we choose a representative for $\gamma\left(t_{1}\right)$. By definition, there is a $\vec{y} \in \mathcal{Y}$ such that

$$
\gamma\left(t_{1}\right)=\left[s_{1}\right], \quad s_{1} \in \mathcal{A}^{\vec{y}} .
$$


Let $B \subset N$ be a neighborhood of $\gamma\left(t_{1}\right)$. We can assume that $B=e(Y)$, where

$$
Y=\left\{\rho_{z, \omega_{0}}\left(S_{0}\right): z \in \omega_{0}\right\} \subset \mathcal{A}^{\vec{y}},
$$

$\omega_{0} \subset \mathbb{R}^{n}$ is open, and

$$
S_{0}=\left(\left(g_{y}\right)_{y \in U}, \omega_{0}\right) \in \mathcal{H}_{\omega_{0}} .
$$

In the next steps $\vec{y}, H=H^{\vec{y}}$ and $B$ are fixed.

Step 7: we know that $\gamma(t) \in R\left(M_{m}\right)$ and $\gamma(t) \in B \subset e\left(A^{\vec{y}}\right)$ for $t=t_{1}-\varepsilon$ when $\varepsilon>0$ is small enough. To combine these facts we show next that $\gamma(t) \in R\left(M^{\vec{y}}\right)$. At first, let $s_{1} \in \mathcal{A}^{\vec{y}}$ be a representation of $\gamma(t)$, that is, $\left[s_{1}\right]=\gamma(t)$ where

$$
s_{1}=\rho_{z_{1}, \omega_{1}}\left(\left(f_{y}^{1}\right), \omega_{1}\right) .
$$

Since $\gamma(t) \in R\left(M_{m}\right)$, there is $\vec{y}_{2}$ and $s_{2} \in R\left(M^{\vec{y}_{2}}\right)$ such that $s_{1} \sim s_{2}$ and

$$
s_{2}=\rho_{z_{2}, \omega_{2}}\left(\left(h_{y} \circ\left(H^{\vec{y}_{2}}\right)^{-1}\right)_{y \in U}, \omega_{2}\right) .
$$

By using the diffeomorphism $F$ in formula (4.10), we see that

$$
h_{y_{j}} \circ\left(H^{\vec{y}_{2}}\right)^{-1}=f_{y_{j}}^{1} \circ F, \quad j=1, \ldots, n .
$$

Since $f_{y_{j}}^{1}\left(z_{1}, \ldots, z_{n}\right)=z_{j}, z=\left(z_{1}, \ldots, z_{n}\right) \in \omega_{1}, j=1, \ldots, n$, where $\vec{y}=\left(y_{1}, \ldots, y_{n}\right)$, this implies

$$
F=H^{\vec{y}} \circ\left(H^{\vec{y}_{2}}\right)^{-1} \quad \text { or } \quad H^{\vec{y}}=F \circ H^{\vec{y}_{2}} .
$$

Since $H^{\vec{y}_{2}}$ is invertible near $\mu(t)$, also $H^{\vec{y}}$ is invertible and it defines regular coordinates at $\mu(t)$. Thus $\gamma(t) \in R\left(M^{\vec{y}}\right)$.

Step 8: next, we show that near $x_{1}=\mu\left(t_{1}\right)$ we can use the coordinates $H=H^{\vec{y}}$. For this it is enough to consider the differential

$$
\mathrm{d} H: \mathrm{T} M_{m} \longrightarrow \mathrm{T} \mathbb{R}^{n}
$$

and show that $\mathrm{d}_{x_{1}} H$ is invertible. Assume that this is not true. Then there is $\xi \in \mathrm{T}_{x_{1}}\left(M_{m}\right)$ such that $\mathrm{d}_{x_{1}} H(\xi)=0$. By Lemma 3.2, there exists $h_{y}, y \in U$, such that $\mathrm{d}_{x_{1}} h_{y}(\xi) \neq 0$.

Let $\left.t=t_{1}-\varepsilon, \varepsilon \in\right] 0, \varepsilon_{1}\left[\right.$ where $\varepsilon_{1}$ is so small that $\gamma(t) \in B$. By step 7 we know that $\gamma(t) \in R\left(M^{\vec{y}}\right)$, that is,

$$
\gamma(t)=\left[\rho_{H(\mu(t)), \omega}\left(\left(h_{y} \circ H^{-1}\right), \omega\right)\right] .
$$

Moreover, $\gamma(t) \in B$ and formula (5.11) imply that

$$
\gamma(t)=\left[\rho_{H(\mu(t)), \omega_{0}}\left(\left(g_{y}\right)_{y \in U}, \omega_{0}\right)\right] .
$$

The relations (5.12) and (5.13) together with (4.11) show that the point $H(\mu(t)) \in \mathbb{R}^{n}$ has a neighborhood $\omega_{t} \subset \mathbb{R}^{n}$ such that

$$
g_{y}(z)=h_{y} \circ H^{-1}(z), \quad z \in \omega_{t}
$$


Let

$$
V=\bigcup_{t \in] t_{1}-\varepsilon_{1}, t_{1}[} H^{-1}\left(\omega_{t}\right) \subset M_{m}
$$

be a neighborhood of the path $\mu(] t_{1}-\varepsilon_{1}, t_{1}[)$. Then

$$
g_{y} \circ H(x)=h_{y}(x), \quad x \in V .
$$

Note that $x_{1}$ is not necessarily in $V$. Let $\xi(t)$ be a smooth vector field along $\left.\left.\mu(] t_{1}-\varepsilon_{1}, t_{1}\right]\right)$ such that $\xi\left(t_{1}\right)=\xi$. Since both sides of (5.14) are continuously differentiable near $x_{1}$, we conclude using the chain rule that

$$
\begin{aligned}
0 & =\mathrm{d}_{H\left(x_{1}\right)} g_{y} \circ \mathrm{d}_{x_{1}} H(\xi) \\
& =\lim _{t \rightarrow t_{1}-} \mathrm{d}_{H(\mu(t))} g_{y} \circ \mathrm{d}_{\mu(t)} H(\xi(t)) \\
& =\lim _{t \rightarrow t_{1}-} \mathrm{d}_{\mu(t)} h_{y}(\xi(t)) \\
& =\mathrm{d}_{x_{1}} h_{y}(\xi) \neq 0,
\end{aligned}
$$

which is a contradiction. Hence $\mathrm{d}_{x_{1}} H$ is invertible implying that $H$ forms regular coordinates in some neighborhood of $x_{1}$. This fact means that the 'wrong' kind of continuations in the case (ii) in Fig. 3 cannot happen either.

Step 9: let us define a germ corresponding to the Green functions at $x_{1}$,

$$
s_{2}=\rho_{z_{2}, \omega_{2}}\left(\left(h_{y} \circ H^{-1}\right), \omega_{2}\right) \in \mathcal{A}^{\vec{y}},
$$

where $z_{2}=H\left(x_{1}\right)$. Let $\omega_{3} \subset \omega_{0} \cap \omega_{2}$ be a connected neighborhood of $H\left(x_{1}\right)$ (see (5.11)) and $\varepsilon$ be small enough. As we saw in step 8 , there is a neighborhood $\omega_{4} \subset \omega_{3}$ of $H\left(\mu\left(t_{1}-\varepsilon\right)\right)$ such that

$$
g_{y}(z)=h_{y} \circ H^{-1}(z)
$$

for $z \in \omega_{4}$ and $y \in U$. Since both sides of Eq. (5.16) are real-analytic functions, this is valid for all $z \in \omega_{3}$. This implies that $\gamma\left(t_{1}\right)$ has a neighborhood in $R\left(M^{\vec{y}}\right) \subset R\left(M_{m}\right)$. Thus $t_{1}$ is an interior point of $K$. Hence $K$ is an open set and $K=[0,1]$. Thus we have shown that $R$ is surjective.

Final step: we have shown that $R$ is a bijection. Since it is continuous and open, it is a homeomorphism.

Proof of Theorem 1.1. - First, we give the proof in the case $\Gamma=\partial M$. By Theorem 5.1 we know $M_{m}$ up to a homeomorphism and the values of the Green functions. By Lemma 2.2, we know the values of the Green function in $M_{m} \backslash M$. Hence we can find the set

$$
R(M)=N \backslash R\left(M_{m} \backslash M\right) .
$$

Thus we can identify $M$ and $R(M)$ by using the homeomorphism $R$. Moreover, we can cover the manifold $N$ with finite number of coordinate neighborhoods $\left(e\left(Y_{i}\right), \Psi_{i}\right)$ given in formula (5.1). These coordinates define a real-analytic structure on $N$ which makes the mapping $R: M_{m} \rightarrow N$ real-analytic diffeomorphism.

Next, we construct the metric on $N$, denote by letters $X=R(x)$ and $Y=R(y)$ the points of $N$ and write $G(X, Y)=G(x, y)$.

Now, the Green function is symmetric in the sense that $G(X, Y)=G(Y, X)$. Therefore we can do continuation respect of parameter $Y$, too. By Lemma 2.2 we know the values of $G(X, Y)$ for each $X, Y \in N \backslash R(M)$. Since $G(X, Y)$ is a real-analytic function of $X$ when $X \neq Y$, we can find $G(X, Y)$ for $X \in N \backslash\{Y\}$ and $Y \in N \backslash R(M)$ by using analytic continuations 
in $\Psi_{i}$-coordinates. By using analytic continuation again and the fact that $G(X, Y)$ is analytic respect to $Y$ when $Y \neq X$, we find $G(X, Y)$ for $X, Y \in N, X \neq Y$.

Finally, we show that the values of Green's functions determine the metric tensor. To keep the notations simple, we identify $M$ and $R(M)$ and construct the appropriate metric tensor from the Green functions.

In dimension $n \geqslant 3$, we see from (3.1) that the Green function has a singularity of the type $c_{n} \mathrm{~d}(x, y)^{-n+2}$, with $c_{n} \neq 0$, when $x$ is near to $y$. Thus by studying the behavior of $G(x, y)$ when $x$ goes to $y$ along a smooth path, we can find the metric tensor at $y$. In two dimensions the Green function has a singularity of the type $c \log \mathrm{d}(x, y)$ with $c \neq 0$. To construct the conformal class of the metric, let us choose some smooth positive measure $\mu$ on $M$. This measure corresponds to the Riemannian volume measure of some unknown metric $\sigma g, \sigma(x)>0$. Then, for any $f \in \mathrm{L}^{2}(M)$, the function

$$
u(x)=\int_{M} G(x, z) f(z) \mathrm{d} \mu(z)
$$

satisfies $\Delta_{\sigma g} u=f$. Let $U \subset M$ be a neighborhood where the pair $h=\left(h_{y_{1}}, h_{y_{2}}\right)$ defines coordinates. By choosing $f$ with $\operatorname{supp}(u) \subset U$ and $v \in \mathrm{C}_{0}^{\infty}(U)$ we can compute the integral

$$
\int_{U} g^{1 / 2} g^{i j} \frac{\partial}{\partial h^{i}} u \frac{\partial}{\partial h^{j}} v \mathrm{~d} h=\left(\Delta_{\sigma g}(u), v\right)_{\mathrm{L}^{2}(M, \sigma g)}=\int_{U} f(z) v(z) \mathrm{d} \mu(z),
$$

where $g_{i j}$ is the metric tensor in the $h$-coordinates. By choosing $f$ and $v$ such that the supports of $u$ and $v$ shrink to one point appropriately, we find the function $g^{1 / 2} g^{i j}$. Thus we can find the conformal class of the metric.

In the case where the Dirichlet-to-Neumann map is given only on a non-empty open subset $\Gamma$ of the boundary we just need a small modification of the arguments in the previous sections. Namely, we define a smooth extension $\widetilde{M}$ by gluing to $M$ a set $V \subset \Gamma \times]-r, 0]$ in the boundary normal coordinates. Then we choose a compact $U \subset V$ and define the pre-sheaf $\mathcal{H}_{\omega}$ as in (4.5) with the additional condition that:

$$
\text { for any } z \in \omega \text { there is } y \in U \text { such that } f_{y}(z) \neq 0 \text {. }
$$

By using the asymptotics of the Green functions near their singularity and the maximum principle, we see that the Green functions are strictly negative in the interior of $M$. This modification implies that the point $x_{1}$ in the formula (5.8) cannot be a boundary point. Otherwise, the proof is analogous to the case $\Gamma=\partial M$. Thus Theorem 1.1 is proven.

\section{Remarks and open problems}

First, we consider a possible extension of Theorem 1.1 part (i).

Remark 6.1. - The method of proof of Theorem 1.1 given above is quite flexible and can be applied to other inverse problems involving a real-analytic structure. We mention, for instance, the problem of finding obstacles inside real-analytic structures. Indeed, a real analytic manifold $M$ with obstacle $D$ can be considered as a manifold $M_{1}=M \backslash D$ where the Dirichlet-toNeumann mapping is given on the known part of the boundary, that is on $\Gamma=\partial M \subset \partial M_{1}$. Hence the construction of the boundary $\partial M_{1}$ gives the boundary $\partial D$ of the obstacle. For other results in this direction, see e.g. [5]. Another likely extension of the result is to piecewiseanalytic Riemannian manifolds. See [6] for the corresponding result in the isotropic case in two dimensions for domains in Euclidean space. 
Remark 6.2. - The method of proof of Theorem 1.1 in two dimensions depends on the fact that we can use local coordinates so that the Green kernel is real-analytic in these coordinates and the fact that the Laplace-Beltrami operator is conformally invariant. In dimension $n \geqslant 3$ the conformally invariant Laplacian is given by

$$
\Delta_{g} u-\frac{n-2}{4(n-1)} R_{g} u=0,
$$

where $R_{g}$ denotes the scalar curvature. Here conformally invariant means that

$$
\left(\Delta_{\sigma g}-\frac{n-2}{4(n-1)} R_{\sigma g}\right)\left(\sigma^{w_{1}} u\right)=\sigma^{w_{2}}\left(\Delta_{g}-\frac{n-2}{4(n-1)} R_{g}\right) u
$$

where $\sigma>0$ and $w_{1}, w_{2}$ are appropriate powers.

We can define the Dirichlet-to-Neumann map $\Lambda_{g}^{c}$ for (6.1) as before under the assumption that 0 is not a Dirichlet eigenvalue for the conformally invariant Laplacian (6.1).

Conjecture 6.1. - Assume that $(M, g)$ is an $n$-dimensional, $n \geqslant 3$, smooth, connected Riemannian manifold with boundary, locally conformal to a real-analytic manifold. Assume that 0 is not a Dirichlet eigenvalue for the conformally invariant Laplacian (6.1). Then $\Lambda_{g}^{c}$ determines a Riemannian manifold $N$ which is conformal to $(M, g)$.

Remark 6.3. - In dimension $n \geqslant 3$ we only used the fact that in local coordinates the Green kernel is real-analytic. Einstein manifolds are real-analytic in harmonic coordinates [9]. Therefore it is natural to conjecture the following result:

Conjecture 6.2. - Assume that $(M, g)$ is an Einstein manifold, $\operatorname{dim}(M) \geqslant 3$. Then $\Lambda_{g}$ determines a Riemannian manifold $N$ which is isometric to $(M, g)$.

\section{REFERENCES}

[1] BROWN R., Global uniqueness in the impedance imaging problem for less regular conductivities, SIAM J. Math. Anal. 27 (1996) 1049-1056.

[2] BROWN R., UhLMANN G., Uniqueness in the inverse conductivity problem for nonsmooth conductivities in two dimensions, Comm. Partial Differential Equations 22 (1997) 1009-1027.

[3] CALDERÓN A.P., On an inverse boundary value problem, in: Seminar on Numerical Analysis and its Applications to Continuum Physics, Soc. Brasileira de Matemática, Río de Janeiro, 1980, pp. 65-73.

[4] Hörmander L., The Analysis of Linear Partial Differential Operators III, Springer-Verlag, 1985.

[5] Is a Kov V., Inverse Problems for Partial Differential Equations, Springer-Verlag, 1998, 284 pp.

[6] KoHN R., Vogelius M., Determining conductivity by boundary measurements II. Interior results, Comm. Pure Appl. Math. 38 (1985) 643-667.

[7] Lee J., Uhlmann G., Determining anisotropic real-analytic conductivities by boundary measurements, Comm. Pure Appl. Math. 42 (1989) 1097-1112.

[8] NaChMAn A., A global uniqueness for a two-dimensional inverse problem, Ann. Math. 142 (2) (1996) 71-96.

[9] Petersen P., Riemannian Geometry, Springer-Verlag, 1998.

[10] Sylvester J., An anisotropic inverse boundary value problem, Comm. Pure Appl. Math. 38 (1990) 201-232.

[11] Sylvester J., Uhlmann G., A uniqueness theorem for an inverse boundary problem, Ann. Math. 125 (2) (1987) 153-169.

[12] TAYLOR M., Partial Differential Equations II, Springer-Verlag, 1996.

[13] TAYLOR M., Partial Differential Equations III, Springer-Verlag, 1996.

[14] Tennison B., Sheaf Theory, Cambridge University Press, 1975, 164 pp.

4 SÉRIE - TOME $34-2001-\mathrm{N}^{\circ} 5$ 
[15] Uhlmann G., Developments in inverse problems since Calderón's foundational paper, in: Harmonic Analysis and Partial Differential Equations, University of Chicago Press, 1999, pp. 245-295 [Essays in honor of Alberto P. Calderón, University of Chicago Press, edited by Christ M., Kenig C. and Sadosky C.].

[16] VekUa I., Generalized Analytic Functions, Pergamon Press, 1962.

(Manuscript received August 13, 2000; accepted, after revision, September 14, 2000.)

Matti LASSAS

Rolf Nevanlinna Institute,

University of Helsinki,

P. O. Box 4,

FIN-00014, Helsinki, Finland

E-mail: Matti.Lassas@helsinki.fi

\section{Gunther UHLMANN}

Department of Mathematics,

University of Washington,

Seattle, WA 98195-4350, USA

E-mail: gunther@math.washington.edu 